\title{
Alineamiento de competencias, actividades y evaluación continuada en grupos grandes. Una propuesta desde el Derecho penal ${ }^{1}$
}

\author{
Alignment of competences, activities and continuous \\ evaluation in large groups. A proposal from criminal \\ law.
}

\section{Joan Baucells Lladós}

Profesor Titular de Universidad

Departament de Ciència Política i de Dret Públic. Àrea de Dret Penal

Universitat Autònoma de Barcelona (UAB), España

E-mail: joan.baucells@uab.cat

\section{Jaume Antich Soler}

Profesor Asociado Laboral

Departament de Ciència Política i de Dret Públic. Àrea de Dret Penal Universitat Autònoma de Barcelona (UAB), España

E-mail: jaume.antich@uab.cat

\section{Antoni Cardona Barber}

Departament de Ciència Política i de Dret Públic. Àrea de Dret Penal Universitat Autònoma de Barcelona (UAB), España

\section{Rosa Carrillo Ruíz}

Investigadora predoctoral

Departament de Ciència Política i de Dret Públic. Àrea de Dret Penal Universitat Autònoma de Barcelona (UAB), España

E-mail: mariarosa.carrillo@uab.cat

\section{María José Cuenca García}

Profesora Agregada Laboral

Departament de Ciència Política i de Dret Públic. Àrea de Dret Penal

Universitat Autònoma de Barcelona (UAB), España

E-mail: mariajose.cuenca@uab.cat

\footnotetext{
${ }^{1}$ El presente estudio es resultado de un proyecto de innovación y de mejora de la calidad docente que, con el mismo título, "Alineament de competències, activitats i el 50\% d'avaluació continuada en grups grans: una proposta des del dret penal”, fue financiado por la Oficina de Qualitat Docent de la Universitat Autònoma de Barcelona en la convocatoria de 2018 (modalidad A).
} 


\title{
Miriam Cugat Mauri
}

Profesora Titular de Universidad

Departament de Ciència Política i de Dret Públic. Àrea de Dret Penal

Universitat Autònoma de Barcelona (UAB), España

E-mail:miriam.cugat@uab.cat

\section{José Riba Ciurana}

Profesor Asociado Laboral

Departament de Ciència Política i de Dret Públic. Àrea de Dret Penal

Universitat Autònoma de Barcelona (UAB), España

E-mail: jose.riba@uab.cat

\section{María José Rodríguez Puerta}

Profesora Titular de Universidad

Departament de Ciència Política i de Dret Públic. Àrea de Dret Penal

Universitat Autònoma de Barcelona (UAB), España

E-mail: maríajose.rodríguez.puerta@uab.cat

\begin{abstract}
Resumen: El presente estudio expone la reflexión realizada por la Unidad de Derecho penal y Criminología de la UAB frente a la exigencia normativa de incrementar hasta el $50 \%$ de la nota final con la evaluación continuada. El reto era visto como una oportunidad para desarrollar resultados de aprendizaje mucho más prácticos; para diseñar e implementar un modelo de evaluación continuada sin que, en contextos de grupos grandes, necesariamente desbordara la capacidad de corrección de los profesores y de retorno de los resultados al alumnado; y, finalmente, para implementar una propuesta colectiva de alineamiento constructivo entre competencias, actividades propuestas a los alumnos dentro y fuera del aula y las actividades de evaluación continuada y final. La propuesta docente fue sometida a una encuesta de los alumnos y un grupo de discusión con los profesores dando como resultado una valoración positiva de los objetivos propuestos, así como de la necesidad de limitar el número de actividades evaluables para mejorar el rendimiento académico de los alumnos.

Palabras clave: evaluación continuada, grupos grandes, alineamiento constructivo.
\end{abstract}

Abstract: The present study exposes the reflection carried out by the Unit of Criminal Law and Criminology of the UAB in front of the normative requirement of increasing up to $50 \%$ of the final grade with the continuous evaluation. The challenge is seen as an opportunity to develop much more practical learning outcomes, to design and implement a continuous assessment model without, in large group contexts, necessarily overflowing the ability of teachers to correct and return of the results to the students and, finally, to implement a collective proposal of constructive alignment between 
competences, activities proposed to the students inside and outside the classroom and the activities of continuous and final evaluation. The proposal was submitted to a student survey and a discussion group with the teachers, resulting in a positive assessment of the proposed objectives as well as the need to limit the number of evaluable activities to improve the academic performance of the students.

Keywords: continuous evaluation, large groups, constructive alignment.

\section{Objeto y delimitación del estudio}

La presente experiencia docente surgió impulsada por un cambio en el modelo docente del grado de Derecho en la UAB. En el curso 2018/2019 su Comisión de Modelo docente propuso implementar que el $50 \%$ de la nota final del alumnado correspondiera a su actividad de evaluación continuada. Esta decisión planteó entre el equipo docente de la Unidad de Derecho penal y Criminología de la UAB una cierta inquietud puesto que había algunos elementos que hacían presagiar dificultades en su implementación. Por un lado, el hecho de que las asignaturas de los grados que se impartían en la Facultad de Derecho fueran de grupos muy numerosos, entre 70-90 alumnos. Por otro lado, que la docencia se impartiera en estructuras académicas donde, en términos generales, no aparecía ninguna subdivisión de los grupos en talleres o seminarios. Finalmente, el nuevo sistema parecía implicar una mayor dedicación de trabajo no solo para el alumno, sino también para un claustro de profesores que vería más que duplicada su dedicación a la corrección y evaluación de las actividades de evaluación continuada.

Lejos de caer en el desánimo, el reto se vio como una oportunidad para poder reflexionar sobre cómo era posible evaluar hasta un 50\% de la nota con actividades de evaluación continuada sin que el profesor tuviera que dedicarse exclusivamente a la corrección de las mismas. Pero, además, también era una oportunidad para replantear colectivamente las metodologías docentes que de forma individual cada uno de nosotros estábamos desarrollando. En concreto, el mayor protagonismo de la evaluación continuada permitiría introducir un mayor peso del trabajo en competencias prácticas, lo que debería permitir mejorar el alineamiento entre estas competencias, las actividades propuestas dentro y fuera del aula y la evaluación de las mismas. 
Para ello, elegíamos la asignatura "Derecho penal III" o "Derecho penal, parte especial" puesto que implicaba algunas ventajas. Se trataba de una asignatura impartida por un número importante de profesores -hasta ocho-, lo que nos permitiría la necesaria y deseada reflexión colectiva. Además, se trataba de una asignatura impartida en diversos grados (Derecho, Criminología, ADE + Derecho) lo que permitiría considerar también distintas variables como el número de alumnos por grupo y la diferencia de organización académica en cada grado ${ }^{3}$. Por último, el aspecto más importante de todos era que esta asignatura se situaba en una fase muy avanzada de la formación de los alumnos en Derecho penal, después de que los alumnos ya hubieran impartido "Derecho penal I", "Derecho penal II" y, en consecuencia, ya tuvieran una sólida base para poder plantearnos actividades más complejas que requirieran habilidades más profesionales.

En definitiva, los principales objetivos del proyecto eran tres:

1. Diseñar e implementar durante el curso 2018/2019 una estrategia y los materiales docentes necesarios para aprovechar el incremento hasta el 50\% de la evaluación continuada para conseguir los resultados de aprendizaje mucho más prácticos del Derecho penal ${ }^{4}$.

2. Diseñar e implementar un modelo de evaluación continuada que pudiera llegar hasta un $50 \%$ de la nota final del alumno sin que, en contextos de grupos grandes, necesariamente desbordara la capacidad de corrección de los profesores y de retorno de la evaluación al alumnado.

\footnotetext{
2 "Derecho penal III" en el Grado de Derecho y la doble titulación de ADE + Derecho y "Derecho penal. Parte especial" en el Grado de Criminología son la misma asignatura. La última obligatoria de Derecho penal donde, después de haber estudiado los aspectos comunes a todos los delitos (libro I del Código penal), se estudia el contenido de los delitos en concreto (libro II del Código penal).

${ }^{3}$ Es importante saber que mientras en el resto de grados de la Facultad de Derecho no es así, en el de Criminología cada grupo teórico (70 alumnos aproximadamente) se subdivide en dos seminarios, de 3035 alumnos aproximadamente.

${ }^{4}$ En realidad, hasta el momento habíamos identificado que muchos de los resultados de aprendizaje previstos en la guía docente de la asignatura tenían cierta dificultad para ser trabajados y evaluados. Piénsese que entre ellos, la guía docente preveía algunos como los siguientes: "Argumentar la aplicación de normas penales en la resolución de problemas penales", "buscar sentencias penales en las bases de datos", "defender oralmente de forma clara y estructurada las ideas", "definir estrategias de defensa de los intereses jurídico penales", "demostrar un razonamiento reflexivo y crítico", "fundamentar la aplicación de normas penales en la resolución de problemas penales", "identificar y valorar la jurisprudencia de las audiencias provinciales y el Tribunal Supremo en materia penal”, "planificar y organizar la gestión del tiempo y recursos", “redactar la resolución de casos penales”, etc.
} 
3. Diseñar e implementar una propuesta colectiva de alineamiento constructivo entre competencias, actividades propuestas a los alumnos dentro y fuera del aula y las actividades de evaluación continuada y final.

\section{Marco teórico. Una propuesta fundamentada sobre el alineamiento} constructivo

El marco teórico fundamental en el que se enmarcaba el proyecto era el alineamiento constructivo (BIGGS, 2005). La idea fuerte de esta propuesta se centra en que una docencia de calidad, que pretenda además motivar al alumnado, requiere que el profesor (2.1) fije objetivos de aprendizaje identificados con competencias profesionales, (2.2) proponga actividades que el alumno sea capaz de desarrollar y (2.3) con un sistema de evaluación alineado con esas actividades y esos objetivos, de manera que se dote de coherencia a los componentes básicos del programa docente (BIGGS, 1996).

\subsection{Los objetivos de aprendizaje deberían estar identificados con competencias} profesionales.

El alineamiento constructivo exige, en primer lugar, que los objetivos de aprendizaje estén bien alineados con las competencias profesionales de la titulación (“justificados”), sean relevantes y ambiciosos ("significativos") y se expresen en términos de conductas indicativas y claras para los alumnos ("claros"). La idea fundamental de esta primera característica es que el alumnado se motive en trabajar la asignatura por su idoneidad en prepararlo para la práctica profesional. El hecho de que trabaje habilidades prácticas nos permite situarnos en un contexto fundamental para el alineamiento constructivo: "lo que hace el estudiante es más importante que lo que hace el profesor" (BIGGS, 2005).

Para ello, la primera sesión de presentación de la asignatura devenía fundamental. En esa sesión al alumno le debía quedar bien claro que las sesiones se dividirán en teóricas y prácticas. En estas segundas es donde se trabajarían las habilidades prácticas orientadas a su profesionalización. El alumno debía asimilar que el objetivo fundamental de esta asignatura, la última del Derecho penal en el grado, serviría para que pudiera plantear y solucionar correctamente un caso penal, como si se tratara de un abogado o magistrado en ejercicio. Además, debería servir para que entendiera que para ello es fundamental "aprender a hacer" cuatro cosas: 1) utilizar la teoría jurídica del delito (parte general del Derecho penal) para plantear y resolver correctamente un caso penal; 2) buscar en las bases de datos y leer comprensivamente antecedentes 
jurisprudenciales del mismo; 3) buscar la opinión al respecto de la doctrina entre los artículos y monografías especializadas; y 4) argumentar jurídico-penalmente la solución en el caso concreto. El reto de aprender a solucionar problemas prácticos, reales y orientados a su profesionalización debería servir para motivar al alumno a trabajar estas cuatro competencias.

Además, para conseguirlo, se les proponía que el último de los problemas penales a resolver sería un caso real que tendrían la oportunidad de conocer asistiendo a una vista oral en la Audiencia Provincial de Barcelona, acercándose a la realidad de la práctica forense y disfrutando con ello (SAIZ GARITAONANDIA, 2011). Si el alumno percibe lo que hacen otros, que ya saben y que se enfrentan a situaciones reales, habiendo experimentado previamente ese quehacer, las posibilidades de poder analizar y evaluar la actividad de los terceros crecen exponencialmente, y el aprendizaje del estudiante se completa satisfactoriamente (DE MIRANDA VÁZQUEZ, 2012).

\subsection{Las actividades propuestas deberían ser asumibles para los alumnos.}

En segundo lugar, en el alineamiento constructivo es también fundamental para la motivación del estudiante que los contenidos y actividades programadas ayuden a los alumnos a lograr los objetivos de aprendizaje de forma eficaz y eficiente. Según la Teoría de los sistemas motivacionales el funcionamiento eficaz depende de variables como la motivación del alumno, sus habilidades, su biología y un entorno sensible (FORD, 1992). Por lo que a las habilidades respecta, esto significa fundamentalmente que las actividades propuestas a los alumnos deben ser de una dificultad que sepan resolver. Por ello era muy importante la calendarización de las mismas desde el principio del curso y, sobre todo, que se tuviera mucho en cuenta la idea de adquisición progresiva de habilidades dirigidas de menor a mayor dificultad a la plena competencia de planteamiento y resolución de un caso penal.

Para ello, se publicaría desde el primer día en el campus virtual de la asignatura la calendarización de las seis sesiones prácticas que coincidirían con la entrega de las seis actividades de evaluación continuada. En la propuesta de estas actividades se tendría en cuenta la necesidad de trabajar de forma individual y progresiva - de menor a mayor dificultad, y de más concreta a más general- las cuatro habilidades destacadas en el epígrafe anterior, empezando por repasar la teoría jurídica del delito como modelo racional de planteamiento y resolución de un caso penal (actividad 1); la búsqueda y lectura comprensiva de un antecedente jurisprudencial (actividad 2); la lectura 
comprensiva de la doctrina (actividad 3 y 5); y la argumentación jurídica de la resolución de casos penales (actividad 4 y 6).

En el caso de la asignatura impartida en el grado de Criminología se introdujo una actividad distinta a sustituir la lectura comprensiva de la doctrina (actividades 3 y 5), consistente en resolver dos casos penales a través de dos juicios simulados. Esta opción se propuso solo en este grado dada la organización de éste en seminarios, lo que permitía mejor la organización y ejecución de esta actividad.

\subsection{Un sistema de evaluación alineado con esas actividades y esos objetivos.}

Por último, la propuesta del alineamiento constructivo entre objetivos, actividades y sistema de evaluación exige que este último refleje claramente los objetivos de aprendizaje como los objetivos a evaluar; los califique mediante criterios objetivos y claros para los alumnos; proporcione retorno a los alumnos de cómo mejorar en su evaluación continuada; y por último, que las actividades propuestas a lo largo del curso permitan al alumno confrontarse con el modelo de examen final o prueba de conjunto.

Para ello, en cada una de las actividades propuestas a los alumnos aparecería en su inicio y reflejados de forma clara cuáles serían los objetivos de aprendizaje de cada una de ellas para que les quedara bien claro cuál sería el objetivo propuesto. En muchas ocasiones, la experiencia nos demuestra cómo los alumnos descubren qué es lo que debían hacer en una actividad en el momento de la corrección, cuando el profesor le explica lo que se esperaba de él. Es decir, cuando ya es demasiado tarde. Además, se les mostraría un ejemplo de resolución correcta de un caso distinto para que conociera exactamente la extensión y tipo de resolución que debería proponer ${ }^{5}$.

En la clase práctica en la que el alumno entregara la actividad debería aprovecharse para generar el retorno suficiente para que pudiera comprobar dónde ha cometido el error, si ha conseguido las habilidades propuestas y cómo mejorar. En la medida, en que las últimas actividades ("la argumentación jurídica de la resolución de un caso penal") actividad 4 y 6- serían más complejas e incluirían las previas, se debería saber trabajar los objetivos anteriores ("aplicación de la teoría jurídica del delito como modelo racional de planteamiento y resolución de un caso penal" -actividad 1- ; "búsqueda y lectura comprensiva de antecedentes jurisprudenciales" -actividad 2-; "búsqueda y lectura comprensiva de doctrina" -actividad 3 y 5-) y el alumno debería conocer

\footnotetext{
${ }^{5}$ Ver en Anexo núm. 1 un ejemplo de actividad 4 y 6 (“Resolución de casos penales") en la que se puede comprobar cómo se detallan los objetivos de aprendizaje propuestos con ella y el ejemplo de resolución.
} 
exactamente cuáles son los elementos de corrección y evaluación de su actividad para que pudiera mejorar las actividades posteriores que, en la lógica de adquisición progresiva de competencias, se irían repitiendo.

Además, el hecho de que las actividades 3 y 5 ("búsqueda y lectura comprensiva de doctrina") se evaluaran a través de una prueba tipo test, permitía al alumno confrontarse hasta en dos ocasiones con el modelo de examen o prueba de conjunto final, que también era un examen tipo test. 


\section{Metodología y resultados esperados. El desarrollo de la experiencia}

Es desde el marco teórico apuntado en el apartado anterior que se propuso la siguiente metodología de trabajo.

Para asegurarnos que las "actividades programadas" ayudarían a los alumnos a lograr los "resultados de aprendizaje prácticos" de forma eficaz y eficiente se propuso una calendarización de hasta seis actividades prácticas que respetaran la idea de adquisición progresiva de habilidades prácticas hasta la plena competencia de planteamiento y correcta resolución de un caso penal. En este sentido se programaron las siguientes seis actividades ordenadas de menor a mayor dificultad y adquisición progresiva de habilidades prácticas de resolución de un caso penal, que fueron publicadas en el campus virtual de la asignatura [ver primera columna de la figura 1]:

\begin{tabular}{|c|c|c|c|}
\hline & $\begin{array}{l}\text { Actividades } \\
\text { programadas }\end{array}$ & Semana & Resultados de aprendizaje prácticos \\
\hline Actividad núm. 1 & $\begin{array}{l}\text { Aplicación de la } \\
\text { teoría jurídica del } \\
\text { delito como } \\
\text { modelo racional de } \\
\text { planteamiento y } \\
\text { resolución de un } \\
\text { caso penal }\end{array}$ & Tercera & $\begin{array}{l}\text { Internalizar la importancia de recuperar } \\
\text { la "teoría jurídica del delito", estudiada } \\
\text { en el curso anterior, como modelo } \\
\text { racional de planteamiento y correcta } \\
\text { solución de un problema penal } \\
\text { (tipicidad, antijuridicidad, culpabilidad, } \\
\text { punibilidad, determinación de la pena, } \\
\text {...) }\end{array}$ \\
\hline Actividad núm. 2 & $\begin{array}{lr}\text { Búsqueda } & \text { de } \\
\text { sentencia } & y \\
\text { elaboración de } & \text { dicha } \\
\text { jurisprudencial } & \end{array}$ & Quinta & $\begin{array}{l}\text { Buscar y leer comprensivamente los } \\
\text { antecedentes jurisprudenciales como } \\
\text { instrumentos para resolver problemas } \\
\text { similares de interpretación y aplicación } \\
\text { de las normas penales. }\end{array}$ \\
\hline Actividad núm. 3 & Lectura doctrinal & Octava & $\begin{array}{l}\text { Buscar y leer comprensivamente } \\
\text { bibliografía especializada para resolver } \\
\text { problemas de interpretación y aplicación } \\
\text { de las normas penales. }\end{array}$ \\
\hline Actividad núm. 4 & $\begin{array}{l}\text { Resolución de los } \\
\text { primeros casos } \\
\text { penales }\end{array}$ & Décima & $\begin{array}{l}\text { En la última de las fases, el alumno debe } \\
\text { aplicar las habilidades desarrolladas } \\
\text { anteriormente a la resolución de un par } \\
\text { de concretos casos penales. }\end{array}$ \\
\hline Actividad núm. 5 & Lectura doctrinal & Decimosegunda & Ídem 3 \\
\hline Actividad núm. 6 & $\begin{array}{l}\text { Resolución de los } \\
\text { segundos } \\
\text { penales }\end{array}$ & Decimocuarta & Ídem 4 \\
\hline
\end{tabular}

Figura 1.

En segundo lugar, al principio de curso deberían quedar bien claros cuáles eran "los resultados de aprendizaje prácticos" de la guía docente, alineados con las competencias profesionales, que querían trabajarse en cada una de las actividades de evaluación continuada [ver tercera columna de la figura 1]. Más allá de identificarlas en la guía docente era importante que aparecieran también identificadas al inicio de cada una de 
las seis actividades propuestas al alumno. De este modo, el alumno se preparaba la actividad sabiendo, de entrada, no solo cuáles eran las habilidades que debía demostrar que sabía hacer en esa concreta actividad, sino también cómo se debían realizar exactamente. En muchas ocasiones, el alumno desconoce con precisión cómo debe presentar la actividad o su extensión, y lo descubre tarde, en el momento de la evaluación o el retorno con el profesor. La propuesta era que se le explicara con anterioridad. Con ello no solo mejoraba la comprensión de lo que tenía que hacer, sino también su rendimiento. Para ello, se colgaría en el campus virtual una rúbrica de solución correcta de un caso similar para cada una de las actividades y se habilitaban los últimos minutos de alguna de las clases teóricas precedentes para presentarlo en el aula ${ }^{6}$.

Finalmente, el sistema de evaluación debía proporcionar retorno a los alumnos de sus errores y calificar su trabajo mediante criterios objetivos y claros para los alumnos que les sirviera para mejorar en sus siguientes actividades. Para ello, se dedicaban cada una de las seis sesiones prácticas, a modo de tutoría grupal, íntegramente a la solución de la actividad propuesta, compartiendo la rúbrica con ellos, esto es, los criterios cuantitativos y cualitativos de cada punto de manera que el alumno en la misma clase pudiera conocer su nota, en qué extremo había fallado y cómo podía mejorar en la siguiente oportunidad. Si cualquier estudiante lo requiriera también podía utilizarse la tutoría individual para conocer los errores cometidos y cómo mejorar en las actividades futuras.

\section{Valoración de la experiencia}

Los resultados del proyecto fueron evaluados a partir del método cuantitativo de "encuesta a los alumnos" de los 5 grupos de Derecho, 1 de ADE + Derecho y 1 de Criminología. Para favorecer la participación se realizó en el aula el día del examen final, justo antes de iniciarlo. De este modo, se consiguió la respuesta de la práctica totalidad de los alumnos participantes (409 encuestas) ${ }^{7}$.

Además, la valoración por parte de los profesores tuvo lugar a través del método cualitativo consistente en una "discusión en grupo". Esta fue realizada al final del

\footnotetext{
${ }^{6}$ Ver en Anexo núm. 1 un ejemplo de modelo de una de las actividades. Cada tipo de actividad tenía su modelo de resolución. En concreto, en el Anexo núm. 1 aparece el de las actividades 4 y 6 "Resolución de los casos penales". Puede observarse como en ella, al margen de fijar claramente "qué" es lo que el alumno debe demostrar que sabe hacer, en los comentarios marginales se aclara al alumno "cómo" se espera de él que dé respuesta a cada uno de los objetivos.

${ }^{7}$ Ver Modelo de encuesta como Anexo 2.
} 
cuatrimestre disponiendo y valorando también el resultado de la evaluación final de los alumnos.

Las principales conclusiones alcanzadas respecto a los tres objetivos propuestos son las siguientes:

\section{Primera: La propuesta docente permitió alcanzar resultados de aprendizaje mucho más prácticos o profesionalizadores.}

Los profesores han manifestado unánimemente que respecto al alcance de competencias prácticas o profesionales

“sí, sí... han ganado. En este tema sí. Quizás merma otros como puede ser el contenido teórico, pero en lo que se refiere a enfrentarse a un caso, enfocarlo, buscar jurisprudencia (...) lo han conseguido" [P3].

"Yo lo que he visto es que los alumnos cuando les has dado más cosas a hacer, más han aprendido y más se han involucrado (...) en comparación con otras asignaturas habían aprendido mucho porque habían hecho esta aplicación práctica” [P4].

La visión de los profesores ha sido corroborada por la de los alumnos. En la pregunta número 1 de la encuesta, los alumnos se han mostrado de acuerdo en que la propuesta de actividades de evaluación continuada les ha "aportado la adquisición de conocimientos útiles para la solución de problemas penales”. El promedio de alumnos evaluados responde con un 3,37 sobre un máximo de 4 a esa pregunta [ver figura 2]. Además, han reconocido en la pregunta 7 que el modelo de evaluación efectivamente valoraba que "un porcentaje de la nota final se base en la aplicación práctica de derecho", siendo éstos los aspectos mejor valorados por los alumnos (también 3,37 sobre un máximo de 4) [ver figura 2]. 


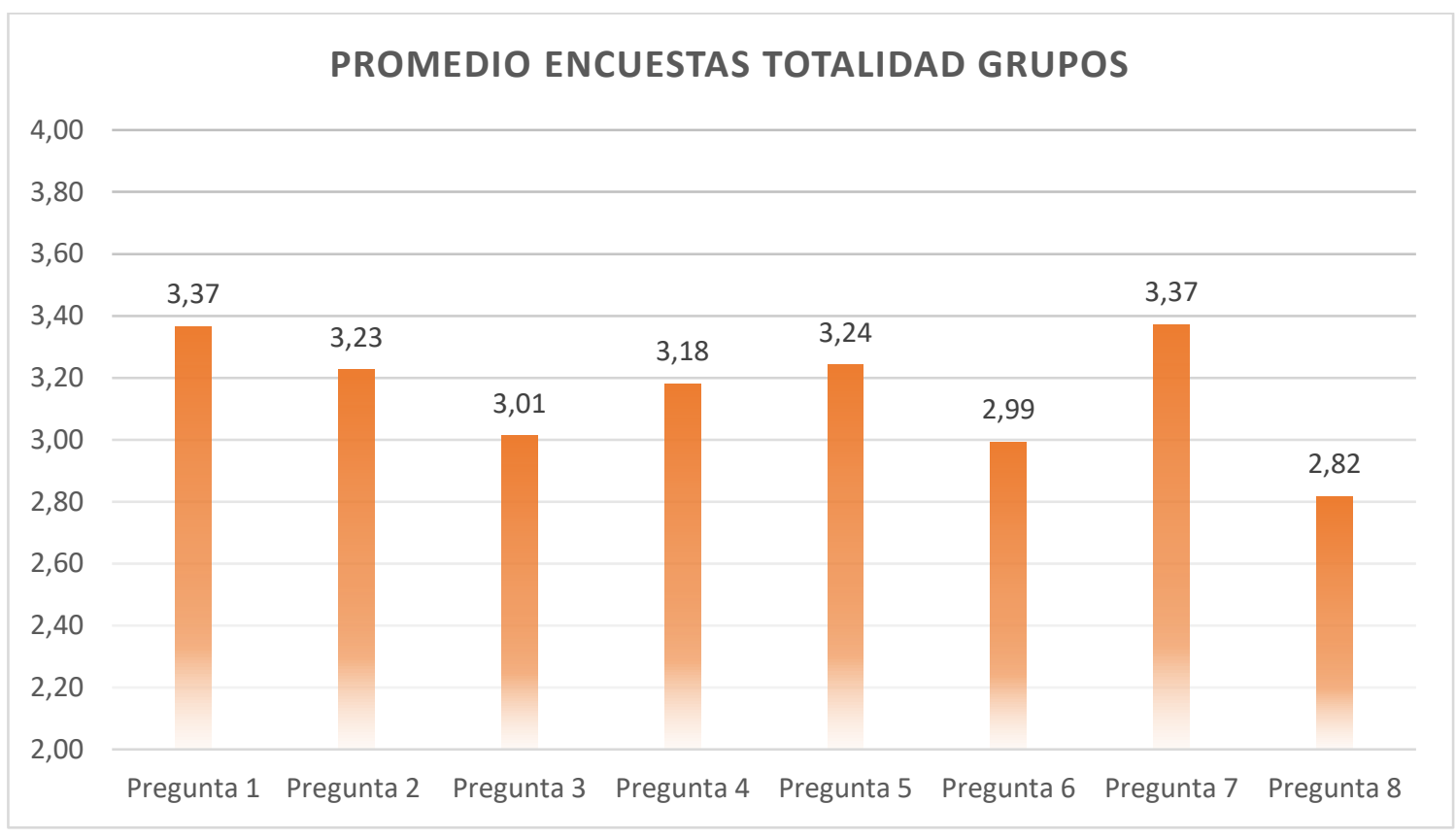

Figura 2.

En consecuencia, creemos que el proyecto ha alcanzado satisfactoriamente el doble objetivo de, por un lado, obtener competencias profesionales de resolución de problemas penales y, de otro, alinear estos objetivos con las actividades realizadas y su evaluación.

Segunda: la evaluación continuada no pudo llegar hasta un $50 \%$ de la nota final del alumno sin que, en contextos de grupos grandes, necesariamente desbordara la capacidad de corrección de los profesores y de retorno de los resultados al alumnado.

Hasta el momento, el peso de la evaluación continuada en la nota final de la asignatura era de un $30 \%$. De este modo, se venían realizando tres actividades consistentes en la resolución de tres casos penales con un valor de hasta 1 punto cada uno. El resto de la nota final, el $70 \%$, provenía de una prueba final de conjunto de contenido fundamentalmente teórico. Como se ha expuesto hasta ahora, en el nuevo contexto, se propuso un total de hasta seis actividades de evaluación continuada. Las dos primeras, de contenido menos dificultoso, con una puntuación de hasta 0,5 puntos cada una ("aplicación de la teoría jurídica del delito como modelo racional de planteamiento y resolución de un caso penal" -actividad 1- ; "búsqueda y lectura comprensiva de antecedentes jurisprudenciales" -actividad 2). Las otras cuatro restantes, con más dificultad, con una puntuación de hasta 1 punto cada una ("búsqueda y lectura 
comprensiva de doctrina" -actividad 3 y 5) y ("la argumentación jurídica de la resolución de un caso penal" -actividad 4 y 6).

De este modo, el total de las actividades de evaluación continuada alcanzaban el $50 \%$ de la nota exigida en la normativa. La propuesta pretendía que, pese a doblar el número de actividades -de 3 a 6-, no supusiera el incremento del tiempo del profesor dedicado a la corrección y evaluación de la misma. La forma de conseguirlo era doble [ver tercera columna de la figura 3]:

\begin{tabular}{|c|c|c|c|c|}
\hline & Contenido & Valor & Corrección & $\begin{array}{l}\text { Labor correctora } \\
\text { del profesor }\end{array}$ \\
\hline Actividad núm. 1 & $\begin{array}{l}\text { Aplicación de la } \\
\text { teoría jurídica del } \\
\text { delito como } \\
\text { modelo racional de } \\
\text { planteamiento y } \\
\text { resolución de un } \\
\text { caso penal }\end{array}$ & 0.5 puntos & $\begin{array}{l}\text { Autocorrección } \\
\text { por parte del } \\
\text { alumno en el aula } \\
\text { a partir de la } \\
\text { rúbrica objetiva } \\
\text { apuntada por el } \\
\text { profesor. }\end{array}$ & NO \\
\hline Actividad núm. 2 & $\begin{array}{lr}\text { Búsqueda } & \text { de } \\
\text { sentencia } & \text { y } \\
\text { elaboración } & \text { de } \\
\text { ficha } & \\
\text { jurisprudencial } & \end{array}$ & 0.5 puntos & $\begin{array}{l}\text { Autocorrección } \\
\text { por parte del } \\
\text { alumno en el aula } \\
\text { a partir de la } \\
\text { rúbrica objetiva } \\
\text { apuntada por el } \\
\text { profesor. }\end{array}$ & NO \\
\hline Actividad núm. 3 & Lectura doctrinal & 1 punto & 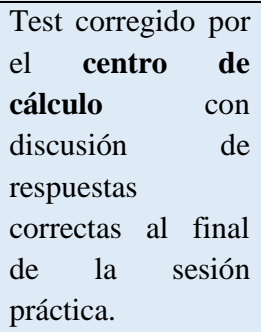 & $\mathrm{NO}$ \\
\hline Actividad núm. 4 & $\begin{array}{l}\text { Resolución de los } \\
\text { primeros casos } \\
\text { penales }\end{array}$ & 1 punto & $\begin{array}{l}\text { Trabajo corregido } \\
\text { fuera del aula por } \\
\text { el profesor } \\
\text { después de la } \\
\text { rúbrica objetiva } \\
\text { comentada en la } \\
\text { clase práctica. }\end{array}$ & SI \\
\hline Actividad núm. 5 & Lectura doctrinal & 1 punto & $\begin{array}{l}\text { Ídem 3: centro de } \\
\text { cálculo }\end{array}$ & $\mathrm{NO}$ \\
\hline Actividad núm. 6 & $\begin{array}{l}\text { Resolución de los } \\
\text { segundos casos } \\
\text { penales }\end{array}$ & 1 punto & Ídem 4: profesor. & SI \\
\hline
\end{tabular}

\section{Figura 3}

De un lado, se introducían las dos primeras actividades, con un valor de 0,5 puntos cada una que se autoevaluaban por el mismo alumno en el aula siguiendo la rúbrica propuesta por el profesor en el aula (“Aplicación de la teoría jurídica del delito" y "Búsqueda de sentencia y elaboración de ficha jurisprudencial'). De este modo, el profesor simplemente debía ofrecer los criterios de corrección en el aula, pero era el mismo 
alumno el que se autoevaluaba y entregaba su nota al final de la sesión. La idea ha sido valorada positivamente por dos profesores, puesto que ha podido comprobar cómo los alumnos eran muy honestos en su autoevaluación:

"Ellos mismos se ponían la nota. A mi me funcionó. Después en el despacho repasé algunos y vi que habían sido muy honestos (...). Se estaban jugando sólo 0,5 puntos y muchos de ellos se suspendieron" [P2];

"Yo sí lo hice. Mantuve las notas que se pusieron ellos" [P3].

o funcionó tan bien, el sistema utilizado y probado en algún grupo consistente en que los alumnos evaluaran las actividades de sus compañeros (SAIZ, M. S. I., GÓMEZ, G. R., RUIZ, M. Á. G., 2012). En este caso, se pudo comprobar cómo les costaba evaluar al compañero, hasta el punto de entregarse algunos trabajos sin evaluar que tuvieron que ser corregidos y evaluados por los responsables docentes.

Por último, la mayoría de profesores por desconfianza las corrigieron ellos mismos con el consiguiente fracaso en el propósito inicial de descargar de labores de corrección a los profesores:

"Por la inexperiencia del primer año, la primera y la segunda práctica las corregimos nosotros" [P1; P4; P5; P6; P7].

De otro lado, las dos actividades consistentes en la "Lectura comprensiva de doctrina" se evaluaban con un examen tipo test de 15 preguntas. Al margen de que este sistema de corrección era evaluado por el Centro de cálculo, sin trabajo extra para el profesor, permitía que el alumno se fuera familiarizando con el examen tipo test que sería el método de la prueba de conjunto final. En consecuencia, y como se podrá observar (ver figura 3), el modelo propuesto pretendía en realidad no solo mantener sino incluso reducir la evaluación por parte del profesor de tres a dos grupos de casos prácticos.

A modo de conclusión, debe destacarse que solo dos profesores se limitaron satisfactoriamente a corregir exclusivamente las dos actividades de "Resolución de casos penales" [P2 y P3] ganando con esto mayores evidencias sobre competencias prácticas de los alumnos, invirtiendo -sin embargo- menor tiempo a la corrección que en el modelo anterior. La mayoría, desconfiando de la autoevaluación de los alumnos, las acabaron corrigiendo ellos mismos asumiendo más carga de corrección de la prevista [P4; P5; P6 y P7]. 
Por último, uno de los profesores valoró:

“en mi opinión, el sistema de evaluación inicialmente propuesto exigía un tiempo de corrección excesivo en comparación con el rendimiento marginal para el alumno, por este motivo, lo modifiqué ligeramente (...) sustituyendo alguna actividad de resolución de casos por la realización de test" [P1].

Tercera: el modelo propuesto ha permitido alcanzar niveles importantes de alineamiento constructivo entre competencias, actividades propuestas a los alumnos dentro y fuera del aula y las actividades de evaluación continuada y final.

Ello es así puesto que el alumno ha podido observar como aquellas competencias claramente profesionales de aplicación práctica del Derecho penal que aparecen en la guía docente aparecían identificadas como objetivos concretos en cada una de las actividades en las que se trabajaban. Además, al no trabajarlas conjuntamente en la solución de casos penales - como se venía haciendo hasta ahora-, sino de forma independiente, como estadios de planteamiento y resolución de los mismos, ha permitido que el alumno se pudiera concentrar mejor en ellas de forma más eficaz y evitando la frustración de no dominar las habilidades requeridas. Finalmente, el retorno de la evaluación continuada en el aula y en la tutoría individual permitía que el alumno supiera como mejorar su evaluación en el futuro puesto que el sistema también le aseguraba tener una segunda oportunidad para desarrollar las habilidades trabajadas.

Si se observan los errores en la actividad "Aplicación de la teoría jurídica del delito como modelo racional de planteamiento y resolución de casos penales" $-1^{\mathrm{a}}$ actividadse podían corregir en las actividades $4^{\mathrm{a}}$ y $6^{\mathrm{a}}$ ("Resolución de un caso penal"). Por su parte, los errores en la actividad "Búsqueda y lectura comprensiva de antecedentes jurisprudenciales" $-2^{\mathrm{a}}$ actividad- se podían enmendar en las actividades $4^{\mathrm{a}}$ y $6^{\mathrm{a}}$ (“Resolución de un caso penal"). Y, por último, los errores en la $3^{\mathrm{a}}$ actividad "Lectura de doctrina" podían resolverse en la actividad $5^{\mathrm{a}}$.

Además, el alumno llegaba a la prueba de conjunto final, que consistía en un examen tipo test, habiéndose enfrentado al mismo tiempo con la respuesta ya a dos exámenes tipo test durante la evaluación continuada: actividades $3^{\mathrm{a}}$ y $5^{\mathrm{a}}$.

En otro orden de consideraciones, con carácter general, los profesores razonaron que:

"la organización previa y conjunta de las prácticas ha tenido dos ventajas claras: a) ha permitido que los estudiantes pudieran organizar su tiempo y esfuerzo desde el primer día, cuando se les proporciona el plan de trabajo y así ha sido reconocido y aplaudido por los 
propios estudiantes, y b) nos ha permitido a los profesores distribuir entre nosotros el trabajo de preparación de las prácticas que con el nuevo sistema se amplía y exige un sistema de evaluación más preciso" [P1].

Ahora bien, en sentido crítico consideraron que el número de actividades -recordemos, hasta seis- era excesivo. En realidad, suponía la realización de alguna actividad cada 15 días. Entre los profesores hubo consenso en que:

“el número de prácticas ha sido excesivo y más para los que las querían realizar con calidad y dedicación. Ante esto, algunos alumnos han optado por entregar prácticas de trámite; otros han llegado a sustraer horas de estudio de la teoría e incluso de asistencia a clase teórica (...)”, [P1].

El incremento de actividades, siempre según los profesores, ha tenido incluso algún impacto en el rendimiento global de la asignatura:

"El caso paradigmático de inoperancia del sistema, para mi ha sido el de una alumna que teniendo muy buen nivel el año pasado (matrícula de honor), esta año ha faltado a muchas clases, ha tenido un rendimiento inferior y ha obtenido una nota peor (...) Retrata fielmente el espíritu del curso, tal y como lo he visto y como me han comunicado los alumnos" [P1].

Contrastando los resultados académicos obtenidos por los alumnos de esta asignatura en este curso respecto el anterior observamos que, en efecto, ha disminuido el número de notables y excelentes ${ }^{8}$, pero debe reconocerse que el modelo ha permitido estimular muy satisfactoriamente, sobre todo, la presentación ${ }^{9}$, pero también el aprobado de los alumnos matriculados ${ }^{10}$ [ver figura 4].

\footnotetext{
${ }^{8}$ En el curso anterior el porcentaje de notables $(30,37 \%)$, excelentes $(2.53 \%)$ y matrículas $(2,02 \%)$ fue sensiblemente superior al curso actual: notables $(28.47 \%)$, excelentes $(1.01 \%)$ y matrículas de honor $(1,77 \%)$.

${ }^{9}$ En un total de 395 alumnos matriculados en esta asignatura en el curso 2017/2018 no se presentaron 16 (el 4,05\% del total), mientras que en el curso objeto de análisis, el 2018/2019 dejaron de presentarse solo 7 (lo que suponía el 1,77\% del total). Un muy buen resultado.

${ }^{10}$ El porcentaje de alumnos con un aprobado en esta asignatura en el curso 2017/2018 fue de 54,68 (216 alumno de 395 totales matriculados), mientras que en el curso objeto de análisis, el 2018/2019, fue el $58,71 \%$ (266 alumnos del total de 453 matriculados).
} 


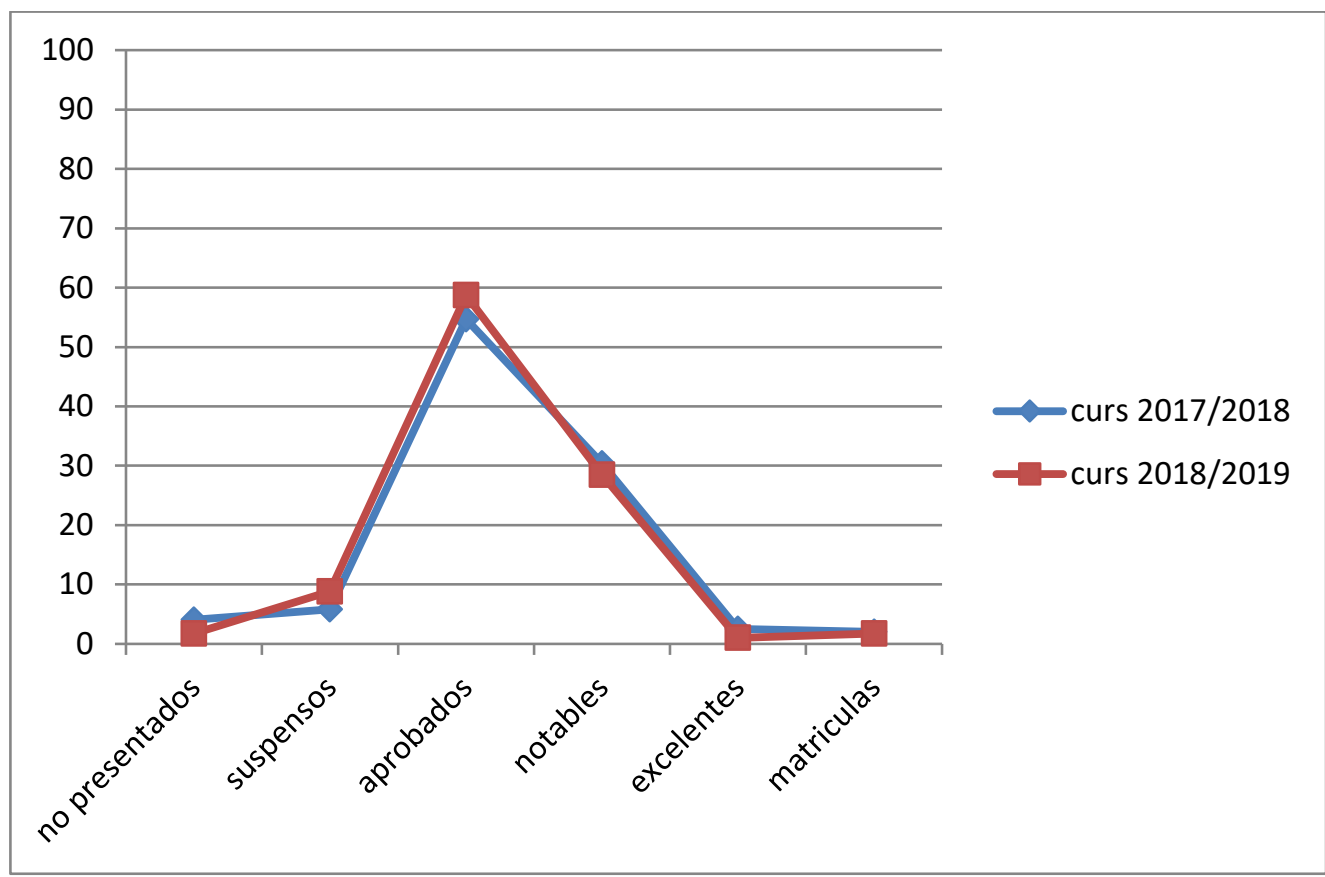

Figura 4

De todos modos, la opinión de los alumnos no es tan contundente como la de los profesores. De un total de 409 alumnos encuestados, solo 81 (el 19,80 \%) propuso reducir el número de actividades en la evaluación. Además, a la pregunta abierta “¿qué modificarías del modelo de evaluación continuada propuesto?" 89 alumnos (el 21,76 \% del total) respondió que no se modificara nada del modelo de evaluación continuada [ver figura 5]. El hecho de que sea la respuesta mayoritaria de los alumnos y que la tercera más votada, con un $9,05 \%$, sea el "no sabe/no contesta" permite concluir que para los alumnos no es un problema el número y la dificultad de las actividades propuestas. Así, expresamente solo 3 alumnos (el 0,73\%) propusieron que las "pruebas sean más fáciles".

\begin{tabular}{|l|l|l|}
\hline $\begin{array}{l}\text { Respuestas a la pregunta: iqué modificarías del modelo de evaluación } \\
\text { continuada? }\end{array}$ & 89 & $21,76 \%$ \\
\hline No Modificación de la Evaluación Continuada & 81 & $19,80 \%$ \\
\hline Menor número de actividades en la evaluación & 37 & $9,05 \%$ \\
\hline NC & 27 & $6,60 \%$ \\
\hline Mayor plazo para la resolución de casos prácticos & 14 & $3,42 \%$ \\
\hline Menor proporción de la evaluación continuada en la nota final & 14 & $3,42 \%$ \\
\hline Más resolución de casos prácticos. & 14 & $3,14 \%$ \\
\hline Mejores indicaciones a la hora de resolver casos & 9 & $2,20 \%$ \\
\hline Realizar la práctica test luego de haber hecho la teoría
\end{tabular}

El resto de respuestas se han desestimado por ser inferiores al 2\% del total de encuestados. 


\section{Figura 5}

Por último, para poder acabar de valorar si el número de actividades propuesto era excesivo, también puede tomarse en consideración, por un lado, las respuestas a la pregunta “¿cuántas horas por semana de promedio has dedicado fuera del aula a las actividades de evaluación continuada? La respuesta media de los siete grupos encuestados fue de 4,87 horas semanales, muy ligeramente por debajo de las 5 horas que deberían dedicar fuera del aula a una asignatura de 6 ECTS con lo que, efectivamente, les quedaba poco tiempo para el estudio. Además, los alumnos reconocen que la evaluación continuada propuesta "ha aumentado la dedicación horaria a la asignatura respecto al resto de las asignaturas de este cuatrimestre", con una respuesta de 2,99 puntos de media, es decir, en parcial acuerdo (pregunta 6) [ver figura 2].

\section{Propuestas de mejora}

En términos generales, el modelo ha sido valorado positivamente por alumnos y profesores por lo que implicaba de mejor organización; mejor trabajo de competencias prácticas o profesionalizadoras; mejor retorno para mejorar en la evaluación continuada y mejor alineamiento de competencias, actividades y evaluación continuada. Sin embargo, como criterios de mejora en el futuro de la planificación docente podemos proponer:

Primero: se deberían limitar las actividades de evaluación continuada a un máximo de cuatro con un triple objetivo. De un lado, ganar más tiempo para que el profesor pueda concluir el programa de la asignatura,

"seis prácticas en el aula han restado tiempo para poder explicar con mayor profundidad determinados temas" [P3].

Y disponer de más tiempo para que los alumnos se prepararan el temario de la asignatura,

"tantas prácticas les sacaba mucho tiempo, y no solo lo sacaban de penal III, tenían más asignaturas, con lo cual les sacaba tiempo para estudiar penal" [P5].

De otro, rebajar la intensidad de entrega de actividades de evaluación continuada por parte de los alumnos pues, tal como demuestran las encuestas, el 6,60\% de los alumnos y la mayoría de los profesores consideraban que era necesario dar un mayor plazo para la resolución de los casos [ver figura 5]. 
Por último, manteniendo el índice de rendimiento de la asignatura, permitir que los buenos alumnos puedan obtener mejores calificaciones.

Segundo: Para ello, se propone eliminar las dos primeras y reducir la evaluación continuada a dos actividades de "Lectura de doctrina" (con un valor de 1 punto cada una) y dos actividades de "resolución de casos penales" (con un valor de 1,5 puntos cada una).

"El contenido de las prácticas ha tenido desniveles. Las dos primeras eran demasiado básicas como para justificar el tiempo y la nota dedicados a ella" [P1].

"Las dos primeras actividades (teoría jurídica del delito y análisis de sentencias), debemos dar por supuesto todo esto. Debemos pensar que gran parte del aula ya lo sabe y si no lo sabe con un repaso de 15 minutos de cómo citar una sentencia, como se busca y cómo resolver un caso (...) porque estas actividades por el poco valor que tienen (0,5 cada una) no vale la pena perder 3 horas de clase)" [P3].

"Las otras cuatro me parecen fantásticas (...) Las lecturas de doctrina y el test correspondiente les obliga a estudiar una parte del temario que no se ha hecho en el aula. El problema es que nos hemos equivocado en escoger los temas porque eran demasiado concretos. Deberíamos introducir más delitos en las lecturas" [P3].

\section{Bibliografía}

BIGGS, J. (1996): Mejoramiento de la enseñanza mediante la alineación constructiva. En: Higher Education, 32, pp. 347-364.

BIGGS, J. (2005): Calidad del aprendizaje universitario. Madrid: Narcea.

DE MIRANDA VÁZQUEZ, C. (2012): "Propuesta de modelo para la docencia del derecho procesal”. En: Revista de Educación y Derecho, 12, pp. 1 a 17

FORD, M. E. (1992): Motivating humans: Goals, emotions, and personal agency beliefs. Thousand Oaks, CA, US: Sage Publications, Inc.

SAIZ GARITAONANDIA, A. (2011): "Uso de múltiples medidas para incentivar la motivación en el aprendizaje del Derecho Procesal Penal". En: J. Picó (Dir.) Aprendizaje del Derecho Procesal: Nuevos retos de la enseñanza universitaria. Barcelona: Editorial J. M. Bosch, pp. 389-396.

SAIZ, M. S. I., GÓMEZ, G. R., RUIZ, M. Á. G. (2012): “La evaluación entre iguales: beneficios y estrategias para su práctica”. En: Revista de educación, 359, pp. 206-231. 
JOAN BAUCELLS LLADÓS ET AL. Alineamiento de competencias, actividades y evaluación continuada en grupos grandes. Una propuesta desde el Derecho penal

\section{Anexo núm. 1: Ejemplo de actividad número 4 y 6 "Resolución de casos penales"}

\begin{abstract}
Pepe, médico de profesión, conocedor que era portador del virus de la Hepatitis B mantuvo varias relaciones sexuales con María, pareja con la que convivía desde hacía 1 año, sin advertirla de que la padecía y sin adoptar precauciones para evitar el contagio. Como consecuencia de las sucesivas relaciones mantenidas con María, ésta contrajo la enfermedad y requirió un tratamiento para su completa curación de 60 dias, 5 de los cuales debióo estar
hospitalizadal.

Criteris d'avaluació. Què han de demostrar els alumnes que saben fer? L'activitat pot arribar a lepresentar fins a 1 punt de la nota final de l'assignatura.
Per tant, el punt (1) no s'obté pel fet d'entregar l'activitat, sinó per realitzar-la de forma excel-lent

Es valorarà especialment que s'assoleixin els següents resultats d'aprenentatge (veure comentaris marginals al document):

a) L'alumne ha de ser capaç d'identificar els fets que poden tenir rellevància penal.

b) L'alumne ha de qualificar correctament els fets des d'un punt de vista penal (quin delicte s'ha comés, dol o imprudència, grau
(a) d'execució, títol de participació dels subjectes actius, identificar -si fos el cas- la presència de causes de justificació, exculpació, ....).

presencia de caplicar la teoria juridica del delicte

Demostrar que sap aplicar
de resolució de casos penals.

d) L'alumne ha d'expressar l'argumentació jurídica que l'ha portat a aquesta solució concreta, fent especial atenció als arguments relacionats amb el cas concret

e) L'alumne ha de demostrar que sap trobar a la base de dades de jurisprudència alguna sentencia per reforçar la seva argumentació. No basta amb citar-la sinó que cal argumentar mínimament per què f) i citar-la correctament a l'escrit.

g) I que sap determinar la pena o penes exactes a imposar.
\end{abstract}

Exemple de solució correcta

Pepe és responsable a títol d'autor directe (art. 28 c.p.) d'un delicte de lesions doloses consumades de l'article $148.4^{\mathrm{a}} \mathrm{C}$ C. I l perguè la víctima tenia una relació d'anàloga afectivitat a la d'esposa amb l'autor doncs era la seva parélla dé fét amb la que convivia des de feia més d'un any. Pel que fa al tipus objectiu ha de constituir el tipus agreujat de l'art. $148.4^{a}$ en relació al tipus bàsic de l'article 147.1 perque d'una banda, (el terme "lesions" abarca també la transmissió d'enfermetats, però en aquest cas concret el tipus agreujat de l'art. 149 no podria aplicar-se perquè el terme "greu lesió somàtica" ha estat reservada a la que "real o potencialment cause un menoscabo de la salud de suma

importància, pues asi lo exige el tratamiente punitivo que equipara ese concepto a las mutilaciones de miembros principales o a la pérdida de sentidos corporales" En aquest tipus d'enfermetat l'excepcional és la mort i el normal és que en un $95-99 \%$ dels casos es produeixi una curació complerta, sense sequeles, com així va succeir en aquest cas [TS (Sala de lo Penal, Sección $1^{\mathrm{a}}$ ) sentencia núm. 242/2013 de 1 abril [RJ 201313185$]$ ]

Pel que fa al tipus subjectiu, el resultat era assumit per l'autor, com a mínim, a través del dol eventuall doncs en la seva condició de metgel coneixia l'alta traves del dol eventuall doncs en la seva condicio de metge coneixia l'alta decidir mantenir, no de forma aillada, sinó de forma reiterada les relacions sexuals amb la seva parella sense informar-li. En els darrers temps el Tribunal sexuals amb la seva parella sense informar-li. En els darrers temps el Tribunal
Suprem s'apropa a la teoría de la probabilitat per delimitar el dol de la Suprem s'apropa a la teoría de la probabilitat per delimitar el dol de la imprudencia conscient. Així admet "la existencia del dolo cuando el autor
somete a la víctima a situaciones peligrosas que no tiene la seguridad de controlar, aunque no persiga el resultado típico. El dolo eventual, por lo tanto no se excluye simplemente por la esperanza de que no se producirá el resultado o porque éste no haya sido deseado por el autor". En un cas similar on es va transmetre la SIDA, el TS aclara que "el acusado, portador del virus $(\mathrm{VIH})$, tenía pleno conocimiento de la posibilidad de que se produjera el resultado de graves lesiones $y$ el alto grado de probabilidad de que realmente se ocasionaran, tras mantener relaciones sexuales con su pareja máxime cuando en dos ocasiones ni siquiera utilizó preservativos. El dolo eventual fluye sin dificultad de los hechos descritos, que impiden la apreciación de una culpa consciente cuyo campo se ve desbordado por el alto grado de probalidad de consciente cuyo campo se descilidad de que se produjeran el contagio cuya representacion resultaba obligada para su mresor, como lo evidencia cl hecho de que conscientemente, y para segur de una enfermedad que se conlagia con ese tipo de relaciones, como as sucedió" [STS (Sala de lo Penal, Sección1a) núm. 1218/2011, de 8 noviembre [RJ 20121284]].

En conseqüència, a Pepe li correspon una pena de presó de dos anys al no concòrrer circumstàncies modificatives de responsabilitat (art. 66.1.6 ${ }^{\mathrm{a}}$ Cं̄ं) juntament amb les penes accesories d'inhabilitació del dret de sufragi passiu durant la condemna (art. 56.1.2 $\mathrm{CP}$ ) i la pena de prohibició d'aproximar-se a la víctima de fins a dos anys (art. $56.2 \mathrm{CP}$ ).

$\left.\left({ }^{*}\right)\right)$

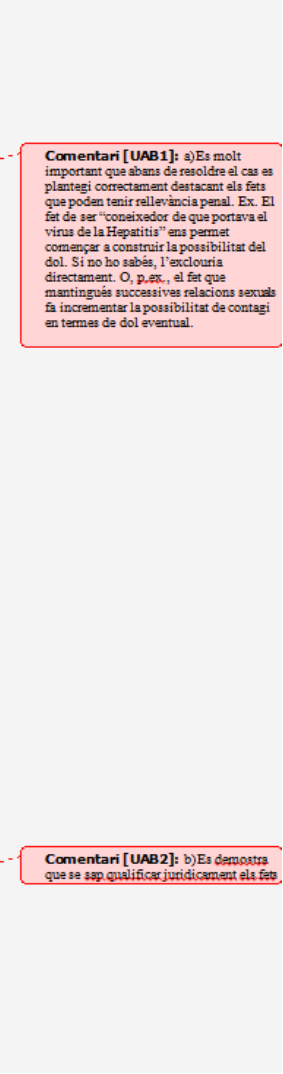

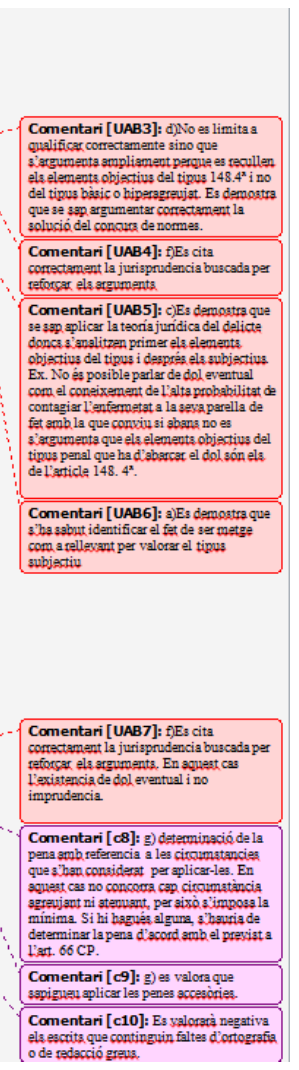




\section{Anexo 2: Modelo de encuesta.}

Encuesta valoración actividad evaluación continuada de la asignatura Derecho penal III. Curso 2018-2019.

Grupo

Este curso has participado en un proyecto de innovación docente en la que se ha planteado una propuesta nueva de evaluación continuada (las seis actividades puntuables realizadas durante el curso). Después de asegurarte que has puesto el número de tu grupo en la parte superior de la hoja, ayúdanos a valorar la experiencia respondiendo a este breve cuestionario de forma absolutamente anónima. Gracias.

Respuestas cerradas:

$\mathbf{1}$ "en total desacuerdo"; $\mathbf{2}$ "en parcial desacuerdo"; 3 "en parcial acuerdo"; 4 "totalmente de acuerdo"

1. La evaluación continuada me ha aportado la adquisición de conocimientos útiles para la solución de problemas penales

2. La evaluación continuada me ha aportado una mejor consolidación de conocimientos teóricos

3. La evaluación continuada ha fomentado el análisis y la autonomía en mi estudio del derecho penal

4. Ha fomentado mi uso de recursos didácticos y fuentes de información (bibliografía, jurisprudencia,...).....

5. Ha aumentado la dedicación horaria a la asignatura respecto a las otras asignaturas de penal de otros cursos

6. Ha aumentado la dedicación horaria a la asignatura respecto al resto de asignaturas de este cuatrimestre

7. Ha permitido que un porcentaje de la nota final se base en la aplicación práctica del derecho .

8. Ha fomentado la motivación para un aprendizaje más constante y personal

Respuestas abiertas:

¿Cuántas horas por semana de promedio has dedicado fuera del aula a las actividades de evaluación continuada?

¿Qué modificarías del modelo de evaluación continuada propuesto? 